ojs.uv.es/index.php/qfilologia/index

Rebut: 23.04.202I. Acceptat: 22.09.202I

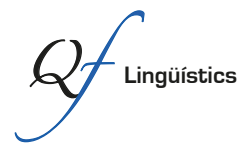

Per a citar aquest article: Novo Arbona, Ainhoa; Peña Fernández, Simón; Eguskiza Sesumaga, Leyre; Jiménez Martín, Eva \& Martínez Odriozola, Lucía. 202I. “¿Vecinas, policías o expertas? Las fuentes informativas en las noticias sobre feminicidios”. Quaderns de Filologia: Estudis Lingüístics XXVI: I93-2II.

doi: $10.7203 / Q F .26 .21984$

\title{
¿Vecinas, policías o expertas? Las fuentes informativas en las noticias sobre feminicidios
}

Neighbors, policemen or experts? The informative sources in the news about femicides

\author{
Ainhoa Novo Arbona \\ Universidad del País Vasco (UPV/EHU) \\ ainhoa.novo@ehu.eus \\ Simón Peña Fernández \\ Universidad del País Vasco (UPV/EHU) \\ simon.pena@ehu.eus \\ Leyre Eguskiza Sesumaga \\ Universidad del País Vasco (UPV/EHU) \\ leyre.eguskiza@ehu.eus \\ Eva JimÉnez MARTÍN \\ Universidad Pública de Navarra (UPNA) \\ eva.jimenez@unavarra.es \\ LuCÍA MarTínez OdRIOZOLA \\ Universidad del País Vasco (UPV/EHU) \\ lucia.martinez@unavarra.es
}

Resumen: El adecuado uso de las fuentes informativas se ha identificado tradicionalmente como uno de los elementos más relevantes a la hora de informar sobre los asesinatos machistas. Sin embargo, en la mayoría de las ocasiones, estos tratamientos informativos se han caracterizado por la escasez de personas expertas que ofrezcan una visión más social del hecho. Este artículo recoge los resultados de una investigación en la que se analizaron 38I informaciones publicadas en los siete principales diarios de Euskadi relativas a los feminicidios ocurridos entre los años 2015 y 2017 y se profundizó con diez entrevistas a personas responsables de medios. Los resultados de la investigación muestran que el vecindario y las familias continúan siendo la fuente informativa más habitual, por delante de las instituciones políticas y las asociaciones feministas, y las fuentes policiales y judiciales. Por su parte, los responsables de los medios de comunicación son conscientes de los peligros del uso del vecindario como fuente 
informativa, y reconocen las dificultades para la correcta cobertura de este tipo de informaciones derivadas de las rutinas periodísticas.

Palabras clave: feminicidios; medios de comunicación; noticias; fuentes informativas; violencia contra las mujeres.

Abstract: The proper use of information sources has traditionally been identified as one of the most relevant elements when reporting on gender-based murders. However, in most cases, these informative treatments have rarely resorted to experts who offer a more social view of the event. This article collects the results of an investigation in which 38I news published in the seven main newspapers of the Basque Country regarding femicides that occurred between 2015 and 2017 were analyzed. Likewise, ten interviews were conducted with media editors. The results of the investigation show that the neighborhood and families continue to be the most common source of information, ahead of political institutions and feminist associations, and police and judicial sources. For their part, media editors are aware of the dangers of using the neighborhood as a source of information, and they recognize the difficulties for the correct coverage of this type of information derived from journalistic routines.

Keywords: femicides; media; news; information sources; violence against women.

\section{Introducción}

Las sociedades con mayores niveles de igualdad y con menor grado de discriminación por causa del género tienden a tener medios de comunicación en los que las noticias sobre la violencia contra las mujeres reflejan el carácter social del problema, en lugar de una visión aislada, privada o pasional de los hechos (Fagoaga, I999).

La transformación que se ha producido en el modo en el que los medios de comunicación cubren las informaciones sobre la violencia contra las mujeres se ha materializado durante las últimas décadas en códigos deontológicos y de autorregulación que establecen una doctrina básica común a todos ellos (Edo \& Zurbano, 20I9; Martínez, 20I2; Sutherland et al., 20I6).

En estos códigos, uno de los puntos recurrentes ha sido la reflexión que se ha realizado sobre el uso de las fuentes informativas (Morgan \& Simons, 2018) pues, según la mayoría de los estudios existentes, los medios han solido recurrir preferentemente a fuentes oficiales en detrimento de personas expertas que ofrezcan una visión más social del hecho (Bullock, 2007; Bullock \& Cubert, 2002; Fagoaga, I994; Menéndez, 20I4; Morgan \& Politoff, 20I2; Zurbano \& Liberia, 2013).

En España, una revisión de los manuales y códigos deontológicos más destacados demuestra el consenso respecto a lo que se considera una buena práctica en el uso de las fuentes a la hora de cubrir la violencia contra las mujeres. 
Así, la mayoría recomienda el uso de fuentes oficiales, personas expertas y asociaciones de mujeres (Zurbano \& García-Gordillo, 20I7). En el caso de Euskadi, 5I medios de comunicación firmaron en febrero de 2016 el Código deontológico $y$ de autorregulación para la publicidad y la comunicación no sexistas (Emakunde, 20I6), que incluye como uno de sus puntos la recomendación de utilizar fuentes expertas y con conocimiento sobre la violencia contra las mujeres.

En este contexto, el objetivo de esta investigación es analizar las fuentes informativas empleadas por los medios de comunicación vascos en los casos de violencia de género con resultado de muerte. En el caso de Euskadi, los estudios existentes en este ámbito son anteriores a la aprobación del Código deontológico (Baraia et al., 20II; Rodríguez, 2008).

La presente investigación no recoge la totalidad de la violencia contra las mujeres o violencia machista. El presente trabajo se engloba en lo que Zurbano, Liberia y Campos consideran el primer nivel definitorio del fenómeno violento, es decir, la violencia del entorno afectivo, en concreto, los actos de violencia perpetrados por hombres a los que la víctima ha estado ligada afectivamente y no la definición más amplia y genérica que hace referencia a la violencia de género que sufren las mujeres por el hecho de ser mujeres que incluirían las agresiones por parte de los hombres sin limitarse al entorno afectivo (20I5). En concreto. esta investigación analiza las fuentes utilizadas en las informaciones publicadas sobre los seis asesinatos machistas ocurridos en Euskadi entre 2015 y 2017 en los que el victimario era la pareja o expareja de la mujer.

Para ello, se plantean las siguientes preguntas de investigación: ¿Cuáles son las fuentes informativas utilizadas en los casos de violencia contra las mujeres con resultado de muerte? (RQI) ¿Cuál es la presencia de las fuentes expertas en estas informaciones? (RQ2) ¿Qué opinión tienen las y los profesionales de los medios de comunicación sobre el uso que realizan de las fuentes en este tipo de informaciones? $\left(\mathrm{RQ}_{3}\right)$.

\section{Marco teórico}

El uso de las fuentes tiene un importante peso en el grado de veracidad que la audiencia otorga a una información. El recurso a más de una fuente identificable (Morgan \& Simons, 20I8) o a fuentes a las que se atribuye autoridad en la materia (Blom, 20I8) contribuyen a generar reacciones más positivas hacia las temáticas que se abordan. 
En el caso específico del uso de fuentes informativas en noticias sobre la violencia contra las mujeres, los estudios existentes subrayan de forma general que se caracterizan por la ausencia de testimonios que contribuyan a una percepción de los hechos como problema social. Así, por ejemplo, en su investigación sobre las informaciones sobre los feminicidios por parte de los medios de comunicación del estado de Utah, Bullock concluye que, a pesar de que la considerable cobertura de los hechos por parte de los medios, la mayoría de las noticias no tenían informantes o habían recurrido únicamente a fuentes oficiales, y no habían contado con personas expertas que pudieran ofrecer una visión más social de la violencia contra las mujeres (Bullock, 2008).

\subsection{Las fuentes policiales y judiciales}

Las fuentes policiales y judiciales han sido tradicionalmente la base principal de las noticias sobre la violencia contra las mujeres, sin distinguir países, ni titularidad, ni tipos de medios (Fernández-Sande et al., 2020). Tal y como reflejan las investigaciones existentes, la policía o el personal de los juzgados han sido el recurso más utilizado por los medios de comunicación para el tratamiento de este tipo de informaciones (Bullock, 2008; Fagoaga, I994, I999; Morgan \& Politoff, 2012; Sutherland et al., 2016).

Sin embargo, el uso recurrente de estas fuentes no resulta inocuo (Bullock $\&$ Cubert, 2002; Gillespie et al., 2013). Así, Meyer (1997) señala que los medios de comunicación las ven como una vía neutral y objetiva a través de la cual narrar la noticia, pero cuestiona su neutralidad. Esta autora sostiene que el posicionamiento sin valoraciones de la perspectiva policial puede resultar engañoso en la medida en la que, desde dicho punto de vista, el crimen se basa en una interacción entre el victimario y la víctima, en la que esta se ve implicada en la explicación de los hechos, lo que deja fuera la visión social y global del problema social (Meyer, I997). También Bullock (2007) considera que la utilización de fuentes oficiales como la policía, los juzgados o personas de la judicatura ofrecen una creencia objetiva a la narración, a pesar de que estas fuentes tienen una posición patriarcal y no feminista.

Por el contrario, Morgan y Simons (20I8) concluyen de sus entrevistas con personas expertas en la cobertura de la violencia contra las mujeres en Australia que la policía ha liderado el cambio de perspectiva del fenómeno. Las políticas destinadas a la formación y sensibilización de los distintos cuerpos 
policiales y judiciales repercuten en el adecuado tratamiento del problema por parte de los medios de comunicación, dado su efecto indirecto y multiplicador como fuentes informativas (Morgan \& Simons, 20I8). En contraposición, las medidas puestas en marcha en Irlanda con el mismo fin para los Gardaí no han parecido suficientes para producir cambios, y se han señalado sus posibles consecuencias en el tratamiento de la violencia machista (Cullen et al., 2019).

El abuso de las fuentes policiales y judiciales tiene como segunda consecuencia la mimetización con la conceptualización y el lenguaje que utilizan, cuyo origen es la legislación vigente, y que puede chocar con la percepción social del problema (Gillespie et al., 2013). El lenguaje judicial tiende a reflejar la categorización de la violencia contra las mujeres como hechos aislados, problemas privados y casos excepcionales. Por ello, la inexistencia de una ley específica sobre violencia contra las mujeres o la adaptación del código penal tendrá una influencia directa sobre la categorización de los hechos por los medios de comunicación.

Por último, la utilización de las fuentes policiales y judiciales para la construcción de la noticia puede influir en el tipo de narración que contribuyen a desarrollar, unos "marcos policiales" (Bullock \& Cubert, 2002) en los que se priorizan "solo los hechos", sin elementos que permitan contextualizarlos ni describir su dimensión social (Cullen et al., 2019).

Tal y como resumen Morgan y Simons (20I8), las críticas al uso recurrente de las fuentes policiales se basan en la presentación de los hechos como fenómenos aislados, para los que la respuesta más adecuada es la ley y el orden sin apelar a la sociedad en su conjunto, lo que promueve su invisibilización.

\subsection{El vecindario}

El vecindario suele percibirse como una fuente de conocimiento fidedigna por su cercanía con la víctima y el victimario, pero en la mayoría de los casos se trata de una fuente desinformada (López-Díez, 2002). Su uso favorece una narración de los hechos en términos de rareza y excepcionalidad, de actos aislados en un contexto de normalidad (Taylor, 2009).

Para los medios de comunicación, una de las ventajas del vecindario como fuente informativa es su accesibilidad, pues son personas abordables desde el momento en el que se llega al lugar de los hechos. Y también pueden contribuir a humanizar la historia, en la medida en que hacen una narración 
en primera persona de los hechos, lo que es más atractivo para la audiencia (Cullen et al., 2019).

Los códigos deontológicos tienden a desaconsejar su uso por el peligro que implica su uso indiscriminado (Martínez, 20I2). A este respecto, el Decálogo para el tratamiento informativo de la violencia contra las mujeres suscrito por los medios de comunicación vascos recoge que puede acudirse a fuentes informantes no expertas (familia, vecindario y testigos), en cuyo caso se usarán esos testimonios "con prudencia y profesionalidad, con el objetivo de no re-victimizar a la mujer o justificar las actuaciones del agresor" (Emakunde, 20I6).

\subsection{Las personas expertas}

De todas las fuentes que pueden emplearse para informar sobre los casos de violencia contra las mujeres, las personas expertas suelen ser las menos utilizadas, a pesar de que a través de ellas es más factible asegurar una visión social de los hechos (Morgan \& Politoff, 20I2). Las personas expertas que participan como fuentes en noticias que abordan un hecho puntual en la mayoría de las veces lo hacen cuando existe una relación previa entre la persona redactora y la experta (Albæk, 20II). Por ello, con el objetivo de facilitar el primer contacto que permita entablar una relación de las personas redactoras con las expertas, algunas instituciones han generado un banco de datos o registro de profesionales y científicas.

Eso sí, la relación de las fuentes expertas con los medios de comunicación no siempre es fácil. Algunas personas expertas han tenido una relación complicada con el periodismo, y la falta de precisión o la omisión de algunos elementos relevantes en la redacción de los artículos suelen ser algunos puntos de tensión entre personas expertas y periodistas. A pesar de ello, las últimas investigaciones señalan un incremento de la frecuencia de la presencia de personas expertas (Shine, 202I).

Desde el punto de vista de las y los profesionales de la información, el tiempo es una cuestión clave para explicar la ausencia de este tipo de fuentes. Las organizaciones especializadas tardan mucho tiempo en dar un posicionamiento sobre la cuestión y eso genera frustración en los periodistas. Además, las personas redactoras deben hacer un esfuerzo para contactar con personas expertas y académicas y esto complica el proceso de redacción (Morgan \& Simons, 20I8). En el caso de España, un estudio de 2017 sobre la cobertura de la violencia contra las mujeres en los medios digitales reflejaba que solamente 
un $8,33 \%$ de las informaciones había consultado con personas expertas u organizaciones (Liberia et al., 2020).

Las oportunidades que ofrece el contacto con este tipo de fuentes transcienden el ámbito de la contextualización. Las organizaciones que trabajan en este ámbito poco a poco van asumiendo que deben estar preparadas para dar una respuesta rápida a estos sucesos, y en el caso concreto de la violencia contra las mujeres, pueden poner en contacto a la persona redactora con alguna mujer que haya vivido situaciones similares proporcionando una visión más humana a la noticia (Cullen et al., 20I9). Así, es más frecuente encontrar a las instituciones de igualdad o expertas feministas en los días posteriores, cuando las noticias abordan las protestas por los asesinatos, pero están menos presentes en las primeras noticias, donde su labor en la contextualización sería recomendable (Menéndez, 20I4).

Si la difícil accesibilidad y el tiempo que emplean para dar una respuesta a los medios son los puntos débiles de estas fuentes, las redes sociales han abierto una ventana de oportunidad, aunque los estudios existentes muestran que se utilizan para obtener los testimonios de personas famosas, deportistas, elite política o personas involucradas en los hechos, y en mucha menos medida de personas expertas (Broersma \& Graham, 2013).

Sin embargo, el uso de una fuente $u$ otra tiene un impacto directo sobre el tipo de narración o marco de interpretación que se está haciendo de los hechos. En su estudio sobre fuentes en la cobertura de asesinatos machistas, Bullock destaca la relevancia de las fuentes para contextualizar de forma adecuada la noticia. Así, citando a Shoemarker y Reese, señala que las fuentes influyen sutilmente en el contenido de la noticia al proporcionar el contexto dentro del cual se evalúa el resto de información contenida en el artículo (Bullock, 2007).

El recurso a cada tipo de fuente también puede influir en el género de los testimonios que se recaban. Cuando se trata de personas expertas, policías o jueces, en la mayoría de los casos son hombres, mientras que encontramos mujeres en el caso de vecinas, amigas íntimas, o compañeras de trabajo tanto de la víctima como del victimario (Carter, I998). La utilización de la policía como fuente principal y en ocasiones, como única fuente y el hecho de que la mayoría de los policías sean hombres contribuye, según Fairbain y Dawson al sostenimiento de la desigualdad (2013).

En resumen, la selección y el uso de las fuentes no solo puede condicionar el modo en el que se contextualizan los sucesos, sino que puede contribuir a generar un tipo de relato (tabla I). Por todo ello, los más recientes códigos 
deontológicos y libros de estilo en los medios de comunicación recomiendan que las informaciones que abordan la violencia contra las mujeres lo hagan desde una perspectiva que incida en su carácter de problema social.

\begin{tabular}{|c|c|c|}
\hline Fuente & Narración & Género informante \\
\hline Policial/judicial & Policial & Hombres \\
\hline Vecindario & Pasional/excepcional & Mujeres \\
\hline Familia y amistades & Estructural/contextualización & Mujeres \\
\hline Personas expertas & Problema social & Hombres \\
\hline
\end{tabular}

Tabla I. Fuentes, género y su efecto en la narración. Fuente: Elaboración propia a partir de Bullock (2007) y Carter (I998)

\section{Metodología}

Para analizar el uso de las fuentes por parte de los medios de comunicación vascos en los casos de violencia de género con resultado de muerte, se ha realizado un análisis de contenido de las informaciones publicadas por los principales diarios de Euskadi sobre los seis feminicidios ocurridos durante los años 20I5, 2016 y 2017.

Los diarios analizados han sido Berria, Deia, Diario de Noticias de Álava, Diario de Noticias de Gipuzkoa, Diario Vasco, El Correo y Gara. Entre todos ellos engloban a nueve de cada diez lectores de la prensa diaria en Euskadi según los datos del Estudio General de Medios (AIMC, 2020). El rastreo de todos estos medios en el periodo de análisis de la investigación permitió identificar un total de 38I piezas, de las cuales únicamente 157 incorporaban al menos una fuente. En el conjunto de las piezas analizadas se han detectado 253 fuentes, dado que algunas noticias incorporaban más de una fuente.

Las fuentes informativas se han clasificado en ocho categorías: fuentes policiales, judiciales, testigos de los hechos, vecindario, familiares y entorno de las víctimas, personas expertas e instituciones del ámbito de la igualdad, e instituciones y representantes políticos.

En relación con el tipo de relato que cada una de ellas puede llegar a favorecer, estas fuentes se han agrupado a su vez en cuatro categorías: relato policial o de sucesos (fuentes policiales y judiciales), relato pasional o excepcional (testigos y vecindario), relato contextual o estructural (familiares y entorno 
de las víctimas) y relato como problema social (personas expertas e instituciones).

Asimismo, para complementar los datos cuantitativos recabados, se han realizado diez entrevistas en profundidad semiestructuradas a personas responsables de la cobertura de estas temáticas en los medios de comunicación vascos (tabla 2). Las entrevistas tuvieron una duración de entre 30 y $45 \mathrm{mi}$ nutos y las preguntas se dirigieron a conocer la valoración sobre el grado de cumplimiento de las recomendaciones del Decálogo. Respecto a la utilización de las fuentes informativas en las noticias sobre feminicidios en sus respectivos medios, se abordaron las siguientes cuestiones: (I) la evolución en su uso, (2) la situación actual, las fuentes más utilizadas y el porqué, (3) las razones para la baja presencia de personas expertas como fuentes, (4) las limitaciones a la hora de elaborar las informaciones y (5) una reflexión crítica sobre sus rutinas de trabajo y sobre algunas de las recomendaciones recogidas en el decálogo.

\begin{tabular}{|c|c|c|c|}
\hline & Medio & Tipo & Puesto \\
\hline E1 & COPE & Radio & Jefa de informativos \\
\hline E2 & eitb.eus & Web & Editora \\
\hline E3 & El Correo & Prensa & Director \\
\hline E4 & ETB & Televisión & Subdirectora de informativos \\
\hline E5 & Radio Euskadi & Radio & Editora de informativos \\
\hline E6 & Onda Cero & Radio & Editor de informativos \\
\hline E7 & Onda Cero & Radio & Redactora de informativos \\
\hline E8 & SER & Radio & Responsable de contenidos \\
\hline E9 & Deia & Prensa & Redactora \\
\hline E10 & RTVE País Vasco & Televisión & Jefe de informativos \\
\hline
\end{tabular}

Tabla 2. Relación de personas entrevistadas

\section{Resultados}

Los resultados muestran, en primer lugar, que las 38I piezas que los diarios vascos de información general han publicado sobre los casos de violencia contra las mujeres con resultado de muerte durante el periodo analizado han 
citado un total de 253 fuentes informativas, es decir, un promedio de 0,66 por información. En total, el 4I,2\% de las noticias recogía explícitamente al menos el testimonio de una fuente directa (figura I).

En términos comparativos entre medios, hay una importante diferencia en la cobertura realizada por las diferentes cabeceras sobre estos casos, que oscila desde las I50 informaciones publicadas por El Correo a las I2 de Berria. En este apartado, parece establecerse una correlación entre el enfoque local con el que se abordan las informaciones sobre violencia contra las mujeres y los ámbitos de difusión de cada uno de los medios.

Si entramos en el detalle del tipo de fuentes utilizadas (tabla 3), podemos observar que el vecindario ha sido la que se ha utilizado con mayor asiduidad (23,7\%). Salvo en el caso de Berria y Gara, son una de las fuentes más habituales en todos los medios analizados.

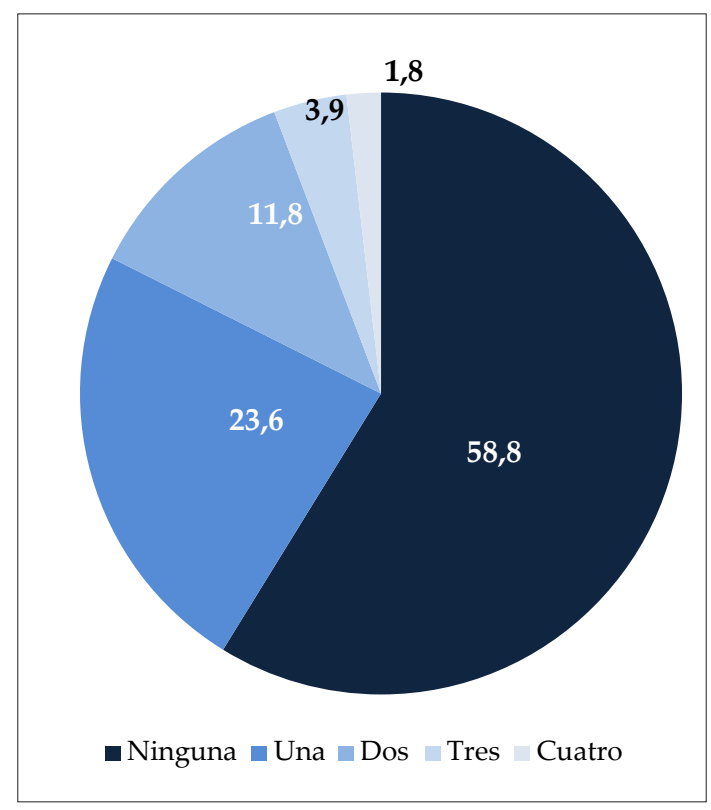

Figura I. Número de fuentes utilizadas en las informaciones (en \%).

Fuente: Elaboración propia

A pesar de que el código desaconseja el uso de estas fuentes, en las entrevistas se refuerza esta tendencia y se cuestiona la norma. Las y los profesionales dan voz directa al vecindario, pero tratan de evitar las expresiones como "era una persona normal" y se cuidan de no blanquear al victimario. Utilizan 
estas fuentes "eliminando todos los testimonios autojustificativos o absurdos de que parecía un buen chico" (EI). Esta es una opinión compartida por otras personas entrevistadas. "En el caso de la violencia de género todos los testimonios de los vecinos van orientados en la misma dirección: era una pareja violenta o era majísima. Me cruzo y siempre me saluda. Eso no aporta nada y puede incluso minimizar el efecto de la noticia, como si fuese consecuencia de un mal rollo personal" (E7). "Sí, se recogen, pero tenemos en la cabeza evitar los cortes que hablen bien del agresor" (E4). "Evitamos siempre [...] esos testimonios de: 'Era muy majo, me cruzaba con él en las escaleras. Yo nunca oí nada, nunca les oí discutir'” (E6).

En todos estos casos, queda presente el debate que se produce en las redacciones de los medios sobre el alcance de esta práctica; "Hoy, por ejemplo, ha ido una persona a cubrir lo de Ollerías y ha hablado con un amigo del agresor que le ha puesto muy bien. Esto ha llevado a una reflexión, a un análisis, a una charla con la persona que estaba allí y a recordar a toda la redacción que tenemos un libro de estilo que contempla que no se debe blanquear al agresor, pero a veces el directo tiene esas fugas" (E5).

Las opiniones sobre el uso del vecindario como fuente, sin embargo, son ambivalentes, y hay quienes defienden que son parte de la historia, y que contribuyen a mostrar que no todo es siempre como parece: "No es cosa de gente rara. Yo estoy convencida de que no sabemos hasta dónde podemos llegar y cualquiera de nuestro entorno puede ser un presunto maltratador... Sin saberlo, incluso" (E3). Las dudas sobre su uso están presentes, por ejemplo, en el caso de tres personas entrevistadas en el lugar de los hechos, de perfil diferente, que señalan que era una persona amable y narran su relación con él: "Es que, a veces, yo me planteo: ¿Y por qué no meterlos?" (Eıo).

En segundo lugar, los medios de comunicación vascos han recurrido a las declaraciones institucionales y testimonios de representantes públicos, en particular el Gobierno vasco y los ayuntamientos de las localidades en las que sucedieron los hechos. En total, estas fuentes institucionales han sumado el 22,I \% de las utilizadas, principalmente como condena y repulsa por los asesinatos.

El entorno familiar y relacional de la víctima es el tercer tipo de fuente más utilizada por los medios de comunicación vascos (18,6\%). En este caso, pueden apreciarse importantes diferencias entre los medios analizados, puesto que para El Correo constituyen la principal fuente de información $(27,4 \%)$, y cuentan también con una importante presencia en las informaciones de 
Diario Vasco. En el resto de los medios analizados, este tipo de testimonios es menos habitual, y no alcanza el i2 \% del total.

El uso de esta fuente genera mayor consenso entre las personas entrevistadas. Algunos medios indican que en ningún caso se ponen en contacto con la familia (EI, E2): "Nosotros buscar el teléfono, no. Vamos y lo que el periodista encuentre allí" (E3). "Si hay posibilidad de algo, pues evidentemente no vas a dar la misma veracidad a un vecino de escalera, que a un familiar directo" (Eio). En estos casos, el testimonio de un familiar es un elemento que hay que considerar a la hora de redactar la noticia, aunque no siempre se incluya en información: "Al final, hay que valorar el testimonio, si procede, o no procede. Si es un testimonio que no me va a aportar nada, y sólo aporta morbo y amarillismo... A mí no me interesa darle morbo, ni amarillismo, a una información de este tipo" (EG).

La familia y el entorno de la víctima pueden ofrecer la oportunidad de empatizar con ella y humanizar la noticia y mostrar la parte trágica de una vida sesgada. "Somos humanos y empatizamos más cuando conocemos las historias... Esa persona puedo ser yo en cualquier momento y si cuento que es una chica que da clases de gimnasia no sé dónde... Si esa puedo ser yo, le está pasando a esta compañera. El protagonista es el hombre, pero para empatizar con la víctima y evitar nuevos casos, tiene importancia contar la historia de vida" (E8). Aunque se tienda a evitar el contacto directo con la familia, el seguimiento del proceso judicial puede ser un buen lugar para recoger estos testimonios. "Bueno, generalmente tú tienes en un juicio [...] lo que va a haber cada día. Entonces, tú, lo miras, y el primer día declara el acusado, la familia de la chica asesinada... Entonces, este día hay que ir" (Eio).

En lo que a las fuentes policiales se refiere -en particular la Ertzaintza y en menor medida las policías municipales- tienen un peso menor en el conjunto de las informaciones $(\mathrm{I} 3,4 \%)$, con detalles sobre los hechos en las primeras etapas informativas.

Todas las personas entrevistadas mencionan a la policía como primera fuente de contacto con los hechos. El departamento de Seguridad del Gobierno vasco, la Ertzaintza o la policía local suelen ser el primer recurso del que se nutren las noticias en el caso de la violencia machista. En esta primera fase, uno de los riesgos que identifican las y los periodistas es que la policía tarda en catalogar los sucesos como violencia machista, lo que puede propiciar una primera narración de los hechos en clave de delitos genéricos que siguen el tono de una narración policial: "Ya sabes cómo funciona. Te dan justo-justo. Parece que les cuesta confirmarte” (EI). Sin embargo, su principal ventaja es 
que se trata de una fuente fidedigna: “Son más creíbles las fuentes policiales que los vecinos" (E3).

Las fuentes expertas, por el contrario, tienen dificultades para abrirse paso en las informaciones. Durante el periodo analizado, constituyen en conjunto 30 de las 253 fuentes utilizadas por los diarios de información general vascos (II,9\%), que solo han recurrido en I2 ocasiones a asociaciones feministas $(4,7 \%)$. En el resto de los casos, se han considerado como fuentes expertas las declaraciones realizadas por el Instituto Vasco de la Mujer - Emakunde $(7, \mathrm{I} \%)$.

Según las personas entrevistadas, una de las aportaciones de Emakunde es atribuir este tipo de crímenes a una situación social estructural: "Te habla alguien de Emakunde, que dice 'pues, lamentablemente, esto es una muestra más de esta sociedad patriarcal, machista” (Eıo). La contribución de las organizaciones feministas o de las instituciones para la igualdad, por tanto, se valora especialmente en la contextualización del hecho como un problema social: "Puede ser por la voz de alguien que lo ha dicho, pero siempre intento que este mensaje esté dentro de la información” (EI). Sin embargo, las personas trabajadoras de los medios apuntan también a la dificultad que tienen en ocasiones para acceder a este tipo de fuentes en el momento preciso de tener conocimiento de los hechos: "Emakunde siempre suele llegar un poco tarde, la verdad” (EI). La accesibilidad y el tiempo son dos obstáculos para una mayor presencia de personas expertas en la cobertura de estos hechos.

Para recabar las opiniones de las fuentes expertas, las redactoras y redactores recurren a las declaraciones de condena por los hechos, las convocatorias de movilización o las ruedas de prensa organizada por estas (Eıo). Aunque algunos medios reconocen no tener un contacto directo con estas organizaciones, otros reconocen tener un directorio de gente y asociaciones a las que acudir en estas temáticas (EI): "Tenemos un directorio de gente... Clara Campoamor [asociación], Emakunde...” (E2).

Para comprender la cobertura realizada por los medios de comunicación, resulta útil también ver la utilización de las fuentes informativas en función del tipo de relato que favorecen.

Así, si agrupamos las fuentes policiales y judiciales, es decir, aquellas que tienen una mayor influencia en la percepción de los hechos como suceso aislado, vemos que suman un total del $18,2 \%$ de las fuentes utilizadas. 


\begin{tabular}{|l|c|c|c|c|c|c|c|c|}
\hline & Berria & Deia & $\begin{array}{c}\text { Diario } \\
\text { Vasco }\end{array}$ & $\begin{array}{c}\text { El } \\
\text { Correo }\end{array}$ & Gara & $\begin{array}{c}\text { Not. } \\
\text { Álava }\end{array}$ & $\begin{array}{c}\text { Not. } \\
\text { Gipuzkoa }\end{array}$ & Total \\
\hline Policía & 7 & 1 & 4 & 16 & 5 & 0 & 1 & 34 \\
\hline Judicial & 1 & 3 & 0 & 4 & 1 & 2 & 1 & 12 \\
\hline Testigos & 0 & 4 & 1 & 4 & 0 & 0 & 2 & 11 \\
\hline Vecindario & 0 & 11 & 9 & 25 & 2 & 5 & 8 & 60 \\
\hline $\begin{array}{l}\text { Familia/ } \\
\text { amistades }\end{array}$ & 0 & 4 & 8 & 29 & 2 & 1 & 3 & 47 \\
\hline Expertas & 0 & 5 & 2 & 12 & 4 & 4 & 3 & 30 \\
\hline Instituciones & 0 & 13 & 9 & 13 & 9 & 4 & 8 & 56 \\
\hline Otros & 0 & 0 & 0 & 3 & 0 & 0 & 0 & 3 \\
\hline Total & 8 & 41 & 33 & 106 & 23 & 16 & 26 & 253 \\
\hline
\end{tabular}

Tabla 3. Tipos de fuentes informativas utilizadas, por medio. Fuente: Elaboración propia

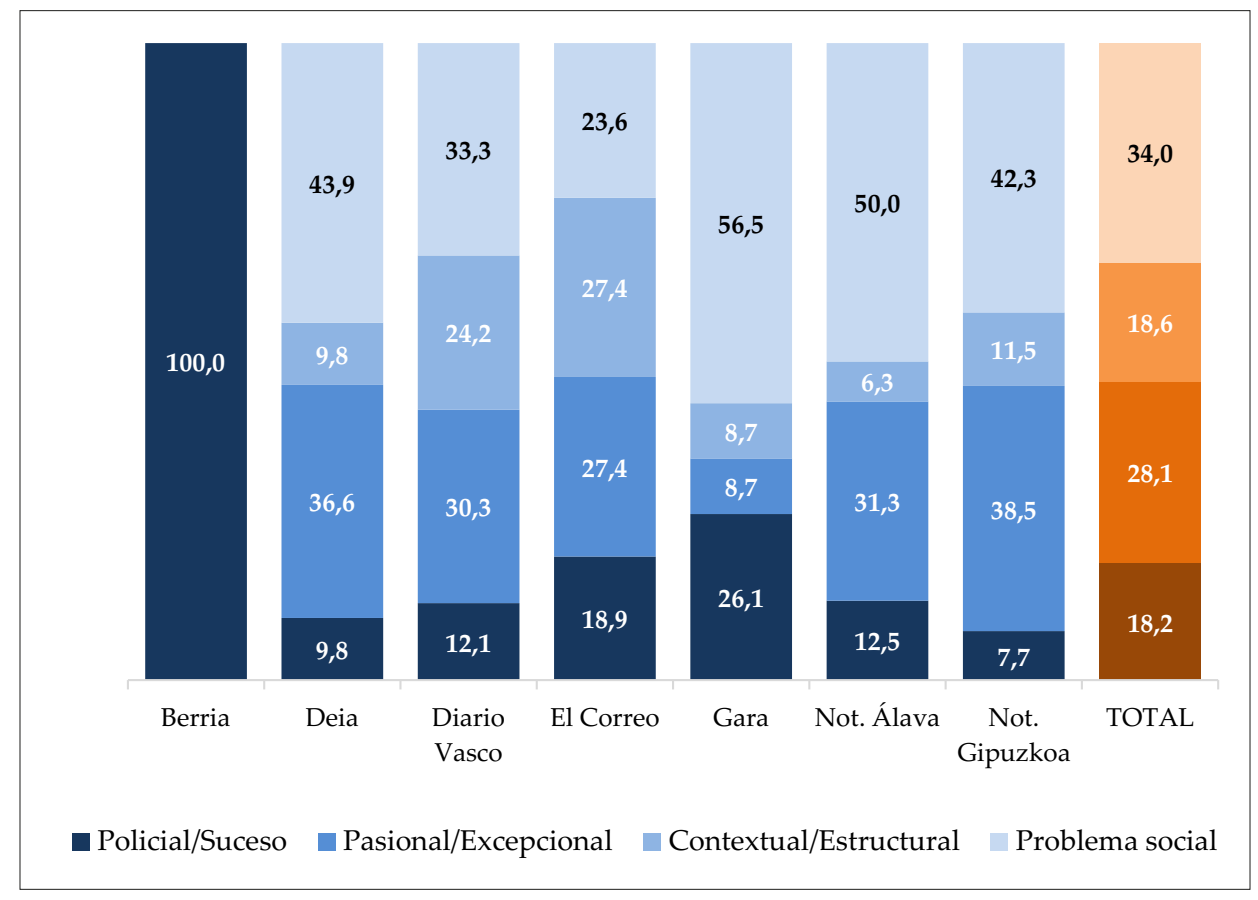

Figura 2. Uso de fuentes por tipo de relato que favorecen (en \%). Fuente: Elaboración propia 
La suma de las declaraciones de las y los testigos de los hechos y el vecindario, por el contrario, alcanzan el 28, $\%$. Este tipo de fuentes se ha vinculado habitualmente con enfoques pasionales y excepcionales de los sucesos, y muchos de los códigos deontológicos desaconsejan su uso. El recurso a este tipo de testimonios es compartido de forma homogénea por todos los medios analizados, salvo Gara y Berria, que apenas las utilizan.

Las declaraciones de familiares y amigos de las víctimas, por su parte, suelen asociarse con un relato contextual de los hechos, que pueden ayudar a visibilizar a la víctima. Este tipo de fuentes alcanza el ı 8,6\% del total, con una presencia particularmente relevante en el caso de El Correo y el Diario Vasco.

Finalmente, el último gran grupo de fuentes lo constituyen aquellas que favorecen un relato de los hechos como problema social. Si agrupamos las declaraciones institucionales -en particular del Gobierno vasco y los ayuntamientos-y los testimonios de las fuentes expertas -asociaciones feministas, Emakunde, etc.- su uso constituye el $34 \%$ de las fuentes analizadas.

\section{Conclusiones y discusión}

El análisis de las fuentes informativas utilizadas en las informaciones publicadas por los diarios de información general sobre los feminicidios ocurridos en Euskadi durante los años 20I5, 2016 y 2017 permite identificar, en primer lugar, un esfuerzo en describir la violencia contra las mujeres como un problema social. Si agrupamos las fuentes analizadas por el tipo de relato que pueden favorecer, el $34 \%$ de los testimonios analizados proceden de instituciones que condenan los hechos o de fuentes expertas que los contextualizan como problema social.

Sin embargo, y a pesar de las indicaciones de los códigos deontológicos (Martínez, 20I2; Sutherland et al., 20I6) y de los propios libros de estilo de los medios de comunicación -que recomiendan que se utilice como fuente con cautela- el vecindario constituye un significativo $23,7 \%$ de los testimonios recabados, en la línea de lo que también muestran otras investigaciones recientes (O’Brien \& Culloty, 2020). Su uso generalizado plantea el riesgo de poder desarrollar un relato pasional de los hechos, que se desarrollen los hechos en términos de excepcionalidad favorable al victimario.

Esta ambivalencia respecto al uso de estas fuentes está incluso recogida en el Decálogo para el tratamiento informativo de la violencia contra las mujeres suscrito por los medios de comunicación vascos, que reconoce que su uso puede 
justificarse por las dificultades que tienen los medios para acceder a datos necesarios para confeccionar este tipo de informaciones en el menor tiempo posible (Emakunde, 2016).

El recurso al vecindario como fuente implica el riesgo de realizar un relato de los hechos en términos de rareza, de actos aislados en un contexto de normalidad (Taylor, 2009). Las y los propios periodistas entrevistados muestran recelos generalizados sobre el uso de este tipo de fuentes e insisten en su peligro y en la cautela con la que hay que emplearlas, pero justifican también su uso por la cercanía que ofrecen y la dificultad de acceder con la premura requerida a testimonios de fuentes expertas, una tendencia que el trabajo para las ediciones digitales de los medios ha agravado aún más.

En efecto, los datos de la investigación muestran una débil presencia de fuentes expertas, un II,9\% del total, a pesar de todas las recomendaciones existentes, y en línea con todas las investigaciones que se han realizado a nivel internacional (Bullock, 2008; Morgan \& Simons, 20I8). Estos datos también son similares a otros estudios en el mismo entorno (Liberia et al., 2020). Su papel clave como contextualizadoras de los hechos y como caracterizadoras del problema social que suponen los asesinatos machistas (Morgan \& Politoff, 20I2) es reconocida y, a la par, demandada por las y los periodistas que elaboran las informaciones. Las organizaciones que trabajan en este ámbito también han percibido la relevancia de reaccionar con rapidez ante estos sucesos (Cullen et al., 2019), y su presencia muestra una leve tendencia al alza (Shine, 202I).

En comparación con otros estudios existentes, los datos de la investigación también ofrecen alguna singularidad. Los estudios en el ámbito anglosajón citan a las fuentes policiales y judiciales como las más citadas en las noticias sobre violencia contra las mujeres (Meyer, I997; Morgan \& Simons, 2018; Cullen et al. 20I9), un lugar que, en el presente estudio, además del vecindario, ocupan las fuentes familiares y el entorno de la víctima (Balica et al., 2020). La mayor presencia en este estudio puede estar asociada a las historias de vida y a la contextualización sobre la víctima, puesto que el objeto de estudio han sido los feminicidios, que tienen una cobertura más extensa y un tratamiento algo diferenciado respecto de la cobertura de otros tipos de violencia contra las mujeres.

Finalmente, ante las lagunas y disfunciones percibidas en el uso de las fuentes informativas, los periodistas mencionan la importancia de una formación práctica en este ámbito (Easteal et al., 202I). Esta necesidad se ve agudizada por el hecho de que, a pesar de que los medios hayan desarrollado 
herramientas para la cobertura de este tipo de informaciones como códigos deontológicos y libros de estilo, sus contenidos no siempre son conocidos por todas las personas que llegan a elaborar estas informaciones ni cubren las múltiples casuísticas que pueden llegar a concurrir en un contexto de elaboración apresurada de las informaciones.

A este respecto, y tal y como puede concluirse del análisis del uso de las fuentes de las noticias y de las opiniones de las personas que las elaboran, la mejora en el tratamiento informativo de la violencia contra las mujeres no puede provenir únicamente de las normas autorreguladoras o de los compromisos institucionales de los medios (Edo y Zurbano, 2019). Más allá del plano teórico, la reflexión sobre el modo efectivo en el que se aplican las recomendaciones, las dificultades y discrepancias que se producen en su uso, y las medidas que pueden tomarse para favorecer su cumplimiento deben acompañar necesariamente a la reflexión y el consenso que impulsan la creación de los códigos deontológicos.

Los procesos de reflexión que se han desarrollado en este ámbito, que han contribuido sin duda a difundir la percepción del problema social de la violencia contra las mujeres, deben aproximarse por lo tanto más a las rutinas periodísticas cotidianas de las y los periodistas para lograr revertir un tipo de cobertura que, a pesar de los avances percibidos, presenta también un margen de mejora.

\section{Bibliografía}

AIMC. 2020. Marco general de los medios en España 2020. Madrid: Asociación para la investigación de los medios de comunicación.

Albæk, Erik. 20II. The Interaction between Experts and Journalists in News Journalism. Journalism I2(3): 335-48.

Balica, Ecaterina; Marinescu, Valentina \& Balica, Mariana. 2020. Was Anastasia Victim of Partner Violence? Media Coverage of Femicide in Romania and Moldova. Journalism Practice. doi: Io.1080/17512786.2020.I799236

Baraia Etxaburu, Gotzon; González García, Sonia; Ancízar Periáñez, Arantza \& De la Sota-Sterling, Rafael. 20II. El tratamiento de la violencia machista en la prensa. Una aproximación a la prensa escrita con difusión en la Comunidad Autónoma del País Vasco. Bilbao: Kualitate.

Broersma, Marcel \& Graham, Todd. 2013. Twitter as News Source: How Dutch and British Newspapers Used Tweets in Their News Coverage, 2007-20II. Journalism Practice 7(4): 446-64. 
Blom, Robin. 20I8. Believing false political headlines and discrediting truthful political headlines: The interaction between news source trust and news content expectancy. Journalism 22(3): 82I-837.

Bullock, Cathy Ferrand \& Cubert, Jason. 2002. Coverage of domestic violence fatalities by newspapers in Washington state. Journal of Interpersonal Violence I7 (5): 475-499.

Bullock, Cathy Ferrand. 2007. Framing domestic violence fatalities: coverage by Utah newspapers. Women's Studies in Communication 30 (1): 34-63.

Bullock, Cathy Ferrand. 2008. Official sources dominate domestic violence reporting. Newspaper Research Journal 29(2): 6-22.

Carter, Cynthia. 1998. When the Extraordinary becomes Ordinary: Everyday news of sexual violence. En Carter, Cynthia; Branston, Gill \& Allan, Stuart (eds.) News, Gender and Power. New York: Routledge, 219-233.

Cullen, Pauline; O'Brien, Anne \& Corcoran, Mary. 20I9. Reporting on Domestic Violence in the Irish Media: An Exploratory Study of Journalists' Perceptions and Practices. Media, Culture \&Q Society 4I(6): 774-90.

Edo Ibañez, Aurora \& Zurbano Berenguer, Belén. 20I9. Deontología periodística en materia de violencias contra las mujeres. ¿Quién elabora los documentos? Un estudio longitudinal desagregado por sexo sobre la autoría. Doxa Comunicación 28: 26I-284.

Easteal, Patricia; Blatchford, Annie; Holland; Kate \& Sutherland; Georgina. 202I. Teaching Journalists About Violence Against Women Best Reportage Practices: An Australian Case Study. Journalism Practice. doi: Io.Io80/ I7512786.2021.I886866

Emakunde - Instituto Vasco de la Mujer. 20I6. Código deontológico y de autorregulación para la publicidad y la comunicación no sexistas. Vitoria-Gasteiz: Emakunde.

Fagoaga, Concha. 1994. Comunicando violencia contra las mujeres. Estudios sobre el mensaje periodístico i: 67-90.

Fagoaga, Concha. 1999. La violencia en medios de comunicación: maltrato en la pareja y agresión sexuada. Madrid: Dirección General de la Mujer.

Fairbairn, Jordan \& Dawson, Myrna. 20I3. Canadian news coverage of intimate partner homicide: analyzing changes over time. Feminist Criminology 8(3): I47-I76.

Fernández-Sande, Manuel; Chagas, Luãn \& Kischinhevsky, Luãn. 2020. Dependencia y pasividad en la selección de fuentes informativas en el periodismo radiofónico en España. Revista española de documentación científica 43(3): 270.

Gillespie, Lane Kirkland; Richards, Tara N.; Givens, Eugena M. \& Smith, M. Dwayne. 2013. Framing Deadly Domestic Violence: Why the Media's Spin Matters in Newspaper Coverage of Femicide. Violence Against Women 19(2): 222-245.

Liberia Vayá, Irene; Zurbano, Belén \& Edo, Aurora. 2020. Femicides in native digital news outlets: greater and better coverage? A study of violence against women in the Spanish media. Observatorio (OBS) I4(I): 45-64.

López Diez, Pilar. 2002. Mujer, Violencia Y Medios de Comunicación. Madrid: Ministerio de Trabajo y Asuntos Sociales. 
Martínez Rodríguez, Beatriz. 20I2. Periodismo y responsabilidad social: el paradigma del tratamiento informativo de la violencia contra la mujer. Vivat Academia XIV (II9): 58-70.

Menéndez Menéndez, María. 20I4. Retos periodísticos ante la violencia de género. El caso de la prensa local en España. Comunicación y sociedad 22: 53-77.

Meyer, Marian. 1997. News Coverage of Violence against Women. Engendering blame. London: Sage.

Morgan, Jenny \& Politoff, Violeta. 20I2. Victorian Print Media Coverage of Violence Against Women: A Longitudinal Study. Melbourne: VicHealth \& University of Melbourne.

Morgan, Jenny \& Simons, Margaret. 20I8. Changing Media Coverage of Violence Against Women. Journalism Practice I2 (9): II65-II82.

O’Brien Anne \& Culloty, Eileen. 2020. Reporting familicide-suicide in broadcast media: An Irish case study to inform better practice. Journalism. doi: Io.II77 /I464884920978028

Rodríguez, María Pilar. 2008. Tratamiento de la violencia de género en la prensa vasca. Cuestiones de género 3: 317-346.

Shine, Kathryn. 202I. Willing but Wary: Australian Women Experts' Attitudes to Engaging with the News Media. Journalism doi: I0.I77/146488492110070

Sutherland, Georgina; McCormack, Angus; Easteal, Patricia; Holland, Kate \& Pirkis, Jane. 20I6. Guidelines for reporting on violence against women in the news media. Australian Journalism Review 38(I): 5-I7.

Zurbano, Belén \& Liberia Vayá, Irene. 20I3. Análisis crítico de los discursos audiovisuales sobre la violencia de género: estudio comparado de caso. Estudios sobre el Mensaje Periodístico I9 (2): 92I-939.

Zurbano Berenguer, Belén; Liberia Vayá, Irene, Campos Mansilla, B. 20I5. Concepto y Representación de la Violencia de Género: Reflexiones sobre el Impacto en la Población Joven. Oñati Socio-legal Series 5(2): 822-845.

Zurbano Berenguer, Belén \& García-Gordillo, Mar. 20I7. Methodological proposal for the evaluation of the ethical quality of news about violence against women. Comunication and Society $30(\mathrm{I}): 73-85$. 
